\title{
A FAMILY OF TRIDIAGONAL PAIRS RELATED TO THE QUANTUM AFFINE ALGEBRA $\mathbf{U}_{q}\left(\widehat{s l_{2}}\right)^{*}$
}

\author{
HASAN ALNAJJAR ${ }^{\dagger}$ AND BRIAN CURTIN ${ }^{\ddagger}$
}

\begin{abstract}
A type of tridiagonal pair is considered, said to be mild of $q$-Serre type. It is shown that these tridiagonal pairs induce the structure of a quantum affine algebra $U_{q}\left(\widehat{s l}_{2}\right)$-module on their underlying vector space. This is done by presenting an explicit basis for the underlying vector space and describing the $U_{q}\left(\widehat{s l_{2}}\right)$-action on that basis.
\end{abstract}

Key words. Leonard pair, Tridiagonal pair, Quantum affine algebra.

AMS subject classifications. 20G42, 15A04, 33D80, 05E35, 33C45, 33D45.

1. Introduction. In [1] the authors study the mild tridiagonal pairs of $q$-Serre type-the main result is a description of the members of this family by their action on an "attractive" basis for the underlying vector space. In this paper we use this action to describe a $U_{q}\left(\widehat{s l_{2}}\right)$-module structure on the underlying vector space of each mild tridiagonal pair of $q$-Serre type. We do so by constructing linear operators on this vector space which essentially satisfy the defining relations for $U_{q}\left(\widehat{s l_{2}}\right)$ in the Chevalley presentation. To state our result precisely we recall some definitions. Throughout this paper, let $\mathcal{F}$ denote a field, and let $V$ denote a vector space over $\mathcal{F}$ with finite, positive dimension. Let $\operatorname{End}(V)$ denote the $\mathcal{F}$-algebra consisting of all $\mathcal{F}$-linear transformations from $V$ to $V$.

Definition 1.1. [5] An ordered pair $A, A^{*}$ of elements from $\operatorname{End}(V)$ is said to be a tridiagonal pair (TDP) on $V$ whenever the following four conditions are satisfied.

(i) Each of $A$ and $A^{*}$ is diagonalizable over $\mathcal{F}$.

(ii) There exists an ordering $V_{0}, V_{1}, \ldots, V_{d}$ of the eigenspaces of $A$ such that

$$
A^{*} V_{i} \subseteq V_{i-1}+V_{i}+V_{i+1} \quad(0 \leq i \leq d),
$$

where $V_{-1}=0, V_{d+1}=0$.

(iii) There exists an ordering $V_{0}^{*}, V_{1}^{*}, \ldots, V_{\delta}^{*}$ of the eigenspaces of $A^{*}$ such that

$$
A V_{i}^{*} \subseteq V_{i-1}^{*}+V_{i}^{*}+V_{i+1}^{*} \quad(0 \leq i \leq \delta),
$$

where $V_{-1}^{*}=0, V_{\delta+1}^{*}=0$.

(iv) There is no proper nonzero subspace $W$ of $V$ such that both $A W \subseteq W$ and $A^{*} W \subseteq W$.

Associated with a TDP are several invariants which we now recall from [5].

* Received by the editors 27 April 2004. Accepted for publication 4 January 2005. Handling Editor: Robert Guralnick.

${ }^{\dagger}$ Department of Mathematics, Private University of Zarka, P.O.Box 2000 - Zarka 13110 Jordan (alnajjar@zpu.edu.jo).

${ }^{\ddagger}$ Department of Mathematics, University of South Florida, 4202 E. Fowler Ave. PHY114, Tampa, FL 33620, USA (bcurtin@math.usf.edu). 
Lemma 1.2. [5] Let $A, A^{*}$ denote a TDP on $V$. The scalars $d, \delta$ from Definition 1.1 are equal; we refer to this common value as the diameter of $A, A^{*}$.

Definition 1.3. Let $A, A^{*}$ denote a TDP on $V$ of diameter $d$. An ordering $V_{0}$, $V_{1}, \ldots, V_{d}$ of the eigenspaces of $A$ is said to be standard whenever it satisfies (1.1). An eigenvalue sequence of $A, A^{*}$ is an ordering $\theta_{0}, \theta_{1}, \ldots, \theta_{d}$ of the eigenvalues of $A$ such that the induced ordering of the eigenspaces of $A$ is standard. An ordering $V_{0}^{*}$, $V_{1}^{*}, \ldots, V_{d}^{*}$ of the eigenspaces of $A^{*}$ is said to be standard whenever it satisfies (1.2). A dual eigenvalue sequence of $A, A^{*}$ is an ordering $\theta_{0}^{*}, \theta_{1}^{*}, \ldots, \theta_{d}^{*}$ of the eigenvalues of $A^{*}$ such that the induced ordering of the eigenspaces of $A^{*}$ is standard.

Lemma 1.4. [5] Let $A, A^{*}$ denote a TDP on $V$ of diameter $d$.

(i) Suppose $V_{0}, V_{1}, \ldots, V_{d}$ is a standard ordering of the eigenspaces of $A$. Then $V_{d}, V_{d-1}, \ldots, V_{0}$ is also a standard ordering of the eigenspaces of $A$, and there are no other standard orderings of the eigenspaces of $A$.

(ii) Suppose $V_{0}^{*}, V_{1}^{*}, \ldots, V_{d}^{*}$ is a standard ordering of the eigenspaces of $A^{*}$. Then $V_{d}^{*}, V_{d-1}^{*}, \ldots, V_{0}^{*}$ is also a standard ordering of the eigenspaces of $A^{*}$, and there are no other standard orderings of the eigenspaces of $A^{*}$.

Lemma 1.5. [5] Let $A, A^{*}$ denote a TDP on $V$ of diameter $d$. Fix standard orderings of the eigenspaces $V_{0}, V_{1}, \ldots, V_{d}$ of $A$ and of the eigenspaces $V_{0}^{*}, V_{1}^{*}, \ldots$, $V_{d}^{*}$ of $A^{*}$. Define $U_{i}(0 \leq i \leq d)$ by

$$
U_{i}=\left(V_{0}^{*}+V_{1}^{*}+\cdots+V_{i}^{*}\right) \cap\left(V_{i}+V_{i+1}+\cdots+V_{d}\right) .
$$

Then $V=U_{0}+U_{1}+\cdots+U_{d}$ (direct sum). The sequence $U_{0}, U_{1}, \ldots, U_{d}$ is called the split decomposition of $V$ relative to the fixed standard orderings. For notational convenience we define $U_{-1}=0$ and $U_{d+1}=0$.

Observe that there are at most four split decompositions of $V$ by Lemma 1.4. Also note that $U_{0}=V_{0}^{*}$ and $U_{d}=V_{d}$.

Lemma 1.6. [5] Let $A, A^{*}$ denote a TDP on $V$ of diameter $d$. Fix standard orderings $V_{0}, V_{1}, \ldots, V_{d}$ of the eigenspaces of $A$ and $V_{0}^{*}, V_{1}^{*}, \ldots, V_{d}^{*}$ of the eigenspaces of $A^{*}$. Then for each $i(0 \leq i \leq d)$, the subspaces $V_{i}, V_{i}^{*}$, and $U_{i}$ have the same dimension-let $\rho_{i}$ denote this dimension. We call the sequence $\rho_{0}, \rho_{1}, \ldots$, $\rho_{d}$ the shape of $A, A^{*}$. The shape is symmetric $\left(\rho_{i}=\rho_{d-i}(0 \leq i \leq d)\right)$ and unimodal $\left(\rho_{i-1} \leq \rho_{i}(1 \leq i \leq d / 2)\right)$. In particular, the shape of $A, A^{*}$ is independent of the choice of standard orderings of the eigenspaces of $A$ and $A^{*}$.

The simplest TDPs are those of shape 1, 1, ., 1, 1. Such TDPs are called Leonard pairs and have been extensively studied $[8,9,10,11,12]$. We consider a mild algebraic generalization of Leonard pair.

Lemma 1.7. [1] Let $A, A^{*}$ denote a TDP on $V$ of diameter d. Let $M$ and $M^{*}$ denote the subalgebras of $\operatorname{End}(V)$ generated by $A$ and $A^{*}$, respectively. Fix standard orderings of the eigenspaces $V_{0}, V_{1}, \ldots, V_{d}$ of $A$ and of the eigenspaces $V_{0}^{*}, V_{1}^{*}, \ldots$, $V_{d}^{*}$ of $A^{*}$. Then the following are equivalent.

(i) $A, A^{*}$ is a Leonard pair.

(ii) $V=M v^{*}$ for some $v^{*} \in V_{0}^{*}$.

(iii) $V=M^{*} v$ for some $v \in V_{d}$.

The preceding result suggests the following generalization of a Leonard pair, from which we exclude the case of Leonard pairs so as to focus on what is new. 
Definition 1.8. With the notation of Lemma 1.7, we say that $A, A^{*}$ is mild whenever $A, A^{*}$ is not a Leonard pair, but $\rho_{0}=\rho_{d}=1$ and $V=M v^{*}+M^{*} v$ for some $v^{*} \in V_{0}^{*}$ and $v \in V_{d}$.

Lemma 1.9. Let $A, A^{*}$ be a mild TDP on $V$ of diameter $d$. Then the shape of $A$, $A^{*}$ is $1,2,2, \ldots, 2,2,1$. Mild TDPs are studied in [1], where an "attractive" basis for the underlying vector space $V$ is constructed. We are also interested in another simplifying property for TDPs. Fix a nonzero scalar $q \in \mathcal{F}$ which is not a root of unity. For any integer $k$ and any nonnegative integer $n$ write

$$
[k]=\frac{q^{k}-q^{-k}}{q-q^{-1}} \quad \text { and } \quad[n] !=[1][2] \ldots[n] .
$$

Definition 1.10. Let $A, A^{*}$ denote a TDP on $V$. Then $A, A^{*}$ is said to be of $q$-Serre type whenever the following hold.

$$
\begin{gathered}
A^{3} A^{*}-[3] A^{2} A^{*} A+[3] A A^{*} A^{2}-A^{*} A^{3}=0, \\
A^{* 3} A-[3] A^{* 2} A A^{*}+[3] A^{*} A A^{* 2}-A A^{* 3}=0 .
\end{gathered}
$$

These relations are called the $q$-Serre relations and are among the defining relations of $U_{q}\left(\widehat{s l_{2}}\right)[2,3]$.

TDPs of $q$-Serre type have been studied in $[1,5,6,7]$. In [1] the authors described the action of a mild TDP of $q$-Serre type on its underlying vector space. We now briefly describe the $U_{q}\left(\widehat{s l_{2}}\right)$-module structure on a mild TDP of $q$-Serre type. We will develop it in more detail in the body of this paper. We begin with some general facts and successively add the $q$-Serre condition and the mild condition. We proceed as in [5]. Given a $\operatorname{TDP} A, A^{*}$ on $V$ and a fixed split decomposition $U_{0}, U_{1}, \ldots$, $U_{d}$ of $V$, for $0 \leq i \leq d$ define $F_{i}: V \rightarrow V$ to be the projection of $V$ onto $U_{i}$. Define $R=A-\sum_{i=0}^{d} \theta_{i} F_{i}$ and $L=A^{*}-\sum_{i=0}^{d} \theta_{i}^{*} F_{i}$. By construction $K R=q^{2} R K$ and $K L=q^{-2} L K$, where $K=\sum_{i=0}^{d} q^{2 i-d} F_{i}$. Observe that $K$ is invertible. By [6], if $A, A^{*}$ is of $q$-Serre type, then $R^{3} L-[3] R^{2} L R+[3] R L R^{2}-L R^{3}=0$ and $L^{3} R-[3] L^{2} R L+[3] L R L^{2}-R L^{3}=0$. In this paper we show that if in addition $A, A^{*}$ is mild, then there exists a linear transformation $\ell: V \rightarrow V$ such that $K \ell=q^{-2} \ell K$ and $\ell R-R \ell=\left(K^{-1}-K\right) /\left(q-q^{-1}\right)$, and there exists a linear transformation $r: V \rightarrow V$ such that $K r=q^{2} r K$ and $r L-L r=\left(K-K^{-1}\right) /\left(q-q^{-1}\right)$. In fact, we give explicit constructions of $\ell$ and $r$. Moreover, we show $\ell$ and $r$ satisfy $\ell L=L \ell, r R=R r$, $r^{3} \ell-[3] r^{2} \ell r+[3] r \ell r^{2}-\ell r^{3}=0$, and $\ell^{3} r-[3] \ell^{2} r \ell+[3] \ell r \ell^{2}-r \ell^{3}=0$. The relations mentioned above for $R, L, r, \ell$ and $K$ are essentially the defining relations for $U_{q}\left(\widehat{s l_{2}}\right)$ in the Chevalley presentation. We now recall this presentation.

DEFINITION 1.11. [2, 3] The quantum affine algebra $U_{q}\left(\widehat{s l_{2}}\right)$ is the associative 
$\mathcal{F}$-algebra with generators $e_{i}^{ \pm}, K_{i}^{ \pm},(i=0,1)$ and relations:

$$
\begin{aligned}
K_{i} K_{i}^{-1} & =K_{i}^{-1} K_{i}=1, \\
K_{0} K_{1} & =K_{1} K_{0}, \\
K_{i} e_{i}^{ \pm} K_{i}^{-1} & =q^{ \pm 2} e_{i}^{ \pm}, \\
K_{i} e_{j}^{ \pm} K_{i}^{-1} & =q^{\mp 2} e_{j}^{ \pm}, i \neq j, \\
{\left[e_{i}^{+}, e_{i}^{-}\right] } & =\frac{K_{i}-K_{i}^{-1}}{q-q^{-1}}, \\
{\left[e_{0}^{ \pm}, e_{1}^{\mp}\right] } & =0,
\end{aligned}
$$

$$
\left(e_{i}^{ \pm}\right)^{3} e_{j}^{ \pm}-[3]\left(e_{i}^{ \pm}\right)^{2} e_{j}^{ \pm} e_{i}^{ \pm}+[3] e_{i}^{ \pm} e_{j}^{ \pm}\left(e_{i}^{ \pm}\right)^{2}-e_{j}^{ \pm}\left(e_{i}^{ \pm}\right)^{3}=0 \quad(i \neq j) .
$$

Our main result is the following.

TheOREM 1.12. Let $A, A^{*}$ be a mild TDP on $V$ of $q$-Serre type with diameter $d \geq 3$. Then $V$ supports a $U_{q}\left(\widehat{s l_{2}}\right)$-module structure on which the following linear operators vanish on $V: e_{1}^{+}-l, e_{0}^{+}-r, e_{1}^{-}-R, e_{0}^{-}-L, K_{0}-K$, and $K_{1}-K^{-1}$. Moreover, this module structure is irreducible.

T. Ito and P. Terwilliger [7] have recently announced that for any TDP of $q$-Serre type there exist linear operators $K, \ell$, and $r$ which together with $R$ and $L$ behave as in Theorem 1.12. The result of Ito and Terwilliger is more general than ours, but in our result we describe the action of the generators on an "attractive" basis. We comment on Theorem 1.12. Finite-dimensional irreducible $U_{q}\left(\widehat{s l_{2}}\right)$-modules are highest weight modules $[2,3]$. It turns out that the weight spaces for the modules in Theorem 1.12 are exactly the subspaces in the split decomposition. Theorem 1.12 identifies raising and lowering operators for the weight spaces of an irreducible $U_{q}\left(\widehat{s l_{2}}\right)$-module with raising and lowering operators associated with a TDP. Thus the $U_{q}\left(\widehat{s l}_{2}\right)$-module structure from Theorem 1.12 is "naturally" related to the given TDP. The operators $r$ and $\ell$ turn out to be the raising and lowering operators associated with a second mild TDP on $V$ of $q$-Serre type with the same split decomposition which is similarly related to $U_{q}\left(\widehat{s l_{2}}\right)$. The reference [7] further elaborates upon these points, so we shall not. In future work, we shall use our construction to describe the associated $U_{q}\left(\widehat{s l_{2}}\right)$-module structure in terms of a tensor product of evaluation modules for $U_{q}\left(\widehat{s l}_{2}\right)[2,3]$.

2. The flat, raising, and lowering maps. We now begin our formal argument. We start by defining and discussing the maps $F_{i}, R, L$.

Theorem 2.1. [5] Let $A, A^{*}$ denote a TDP on $V$ with diameter d. Fix standard orderings of the eigenspaces of $A$ and $A^{*}$, and let $U_{i}(0 \leq i \leq d)$ denote the corresponding split decomposition of $V$. Then the following hold.

(i) $V=U_{0}+U_{1}+\cdots+U_{d}$ (direct sum).

(ii) $\left(A-\theta_{i} I\right) U_{i}=U_{i+1}(0 \leq i \leq d)$.

(iii) $\left(A^{*}-\theta_{i}^{*} I\right) U_{i}=U_{i-1}(0 \leq i \leq d)$. 
Definition 2.2. [5] With the notation of Theorem 2.1, let $F_{i}: V \rightarrow V(0 \leq i \leq$ d) denote the linear transformations that satisfy the following.

(i) $\left(F_{i}-I\right) U_{i}=0(0 \leq i \leq d)$.

(ii) $F_{i} U_{j}=0$ if $i \neq j(0 \leq i \leq d)$.

Observe that $F_{i}$ is the projection map from $V$ onto $U_{i}$.

Lemma 2.3. [5] With reference to Definition 2.2, the following hold.

(i) $F_{i} F_{j}=\delta_{i j} F_{i}(0 \leq i, j \leq d)$.

(ii) $\sum_{i=0}^{d} F_{i}=I$.

(iii) $F_{i} V=U_{i}(0 \leq i \leq d)$.

DEFInITION 2.4. With reference to Definition 2.2, set

$$
R=A-\sum_{i=0}^{d} \theta_{i} F_{i}, \quad L=A^{*}-\sum_{i=0}^{d} \theta_{i}^{*} F_{i}
$$

LEMMA 2.5. [5] With reference to Definition 2.4, for $0 \leq i \leq d$ and for $v \in U_{i}$,

$$
R v=\left(A-\theta_{i} I\right) v \quad \text { and } \quad L v=\left(A^{*}-\theta_{i}^{*} I\right) v .
$$

Corollary 2.6. [5] With reference to Definition 2.4,

$$
R U_{i} \subseteq U_{i+1} \quad \text { and } \quad L U_{i} \subseteq U_{i-1} \quad(0 \leq i \leq d) .
$$

The maps $R$ and $L$ are referred to as the raising and lowering maps with respect to the split decomposition because of the behavior described in Corollary 2.6. We conclude this section by introducing a linear transformation $K$.

Definition 2.7. With reference to Definition 2.2 , set $K=\sum_{i=0}^{d} q^{2 i-d} F_{i}$. Note that $K$ is invertible with inverse $K^{-1}=\sum_{i=0}^{d} q^{d-2 i} F_{i}$.

COROLlary 2.8. With reference to Definitions 2.4 and 2.7,

$$
K R=q^{2} R K \quad \text { and } \quad K L=q^{-2} L K .
$$

Proof. Straightforward.

3. Some relations for $R$ and $L$. In this section we recall that if $A, A^{*}$ is a TDP of $q$-Serre type, then $R$ and $L$ satisfy the $q$-Serre relations.

Lemma 3.1. [5] Let $A, A^{*}$ denote a TDP on $V$ of $q$-Serre type and diameter $d$. Then there exist eigenvalue and dual eigenvalue sequences which satisfy

$$
\theta_{i}=q^{2 i} \theta, \quad \theta_{i}^{*}=q^{2 d-2 i} \theta^{*} \quad(0 \leq i \leq d)
$$

for some nonzero scalars $\theta, \theta^{*} \in \mathcal{F}$.

LEMma 3.2. With reference to Definitions 2.2 and 2.7, assume that the eigenvalue and dual eigenvalue sequences satisfy (3.1). Then

$$
A=q^{d} \theta K+R \quad \text { and } \quad A^{*}=q^{d} \theta^{*} K^{-1}+L .
$$


Proof. Clear from Definitions 2.4 and 2.7.

Lemma 3.3. [5] Let $A, A^{*}$ be a TDP of q-Serre type, and let $R$ and $L$ be as in Definition 2.4. Then

$$
\begin{aligned}
& R^{3} L-[3] R^{2} L R+[3] R L R^{2}-L R^{3}=0 \\
& L^{3} R-[3] L^{2} R L+[3] L R L^{2}-R L^{3}=0 .
\end{aligned}
$$

4. Mild TDPs of $q$-Serre type. In this section we recall some results from [1] concerning mild TDPs of $q$-Serre type.

Notation 4.1. Let $A, A^{*}$ denote a mild TDP on $V$ of $q$-Serre type with diameter $d \geq 3$. Fix standard orderings of eigenspaces of $A$ and $A^{*}$ such that (3.1) holds. Fix a nonzero vector $v^{*} \in U_{0}$.

Lemma 4.2. [1] With Notation 4.1, fix any nonzero scalar $\alpha \in \mathcal{F}$. Define

$$
\begin{aligned}
v_{i}^{*} & =\left(A-\theta_{i-1} I\right) \ldots\left(A-\theta_{1} I\right)\left(A-\theta_{0} I\right) v^{*} \quad(0 \leq i \leq d), \\
v_{i} & =\alpha\left(A^{*}-\theta_{i+1}^{*} I\right) \ldots\left(A^{*}-\theta_{d-1}^{*} I\right)\left(A^{*}-\theta_{d}^{*} I\right) v_{d}^{*} \quad(1 \leq i \leq d) .
\end{aligned}
$$

Then the following hold.

(i) $v_{0}^{*}$ is basis for $U_{0}$.

(ii) For $1 \leq i \leq d-1$, the pair $v_{i}^{*}$, $v_{i}$ is a basis for $U_{i}$.

(iii) $v_{d}=\alpha v_{d}^{*}$ is a basis for $U_{d}$.

Moreover, $v_{0}^{*}, v_{1}, v_{1}^{*}, v_{2}, v_{2}^{*}, \ldots, v_{d-1}, v_{d-1}^{*}, v_{d}$ is a basis for $V$.

LEMMA 4.3. With reference to Notation 4.1, let $v_{i}(1 \leq i \leq d)$ and $v_{i}^{*}(0 \leq i \leq$ $d-1$ ) be as in Lemma 4.2. Then

$$
\begin{array}{ll}
K v_{i}=q^{2 i-d} v_{i} & (1 \leq i \leq d), \\
K v_{i}^{*}=q^{2 i-d} v_{i}^{*} & (0 \leq i \leq d-1) .
\end{array}
$$

Proof. Immediate from the definition of $K$ and Lemma 4.2. $\square$

REMARK 4.4. In [1] the authors describe the action of $A, A^{*}$ on the basis of Lemma 4.2 in terms of six parameters $\theta, \theta^{*}, q, \lambda, \mu$, and $\mu^{*}$. Furthermore, it was shown in [1] that $\mu=\mu^{*}$ if and only if the scalar $\alpha$ in Lemma 4.2 satisfies $\alpha[d-1] ![d] ! \lambda^{d-2} \mu=$ [2]. From now on we assume that $\alpha$ satisfies this condition.

TheOREM 4.5. [1] With reference to Notation 4.1, let $v_{i}(1 \leq i \leq d), v_{i}^{*}(0 \leq$ $i \leq d-1)$ be as in Lemma 4.2. Then there exist nonzero $\lambda, \mu \in \mathcal{F}$ such that

$$
\begin{aligned}
R v_{i}^{*} & =v_{i+1}^{*} \quad(0 \leq i \leq d-2), \\
R v_{d-1}^{*} & =\gamma_{d} \mu v_{d}, \\
R v_{i} & =\lambda_{i} v_{i+1}+\gamma_{d-i} \mu v_{i+1}^{*} \quad(1 \leq i \leq d-2), \\
R v_{d-1} & =\left(\lambda_{d-1}+\gamma_{d-1} \mu^{2}\right) v_{d}, \\
R v_{d} & =0
\end{aligned}
$$


where

$$
\begin{aligned}
\lambda_{i} & =[i][d-i] \lambda \quad(1 \leq i \leq d-1), \\
\gamma_{i} & =\frac{[i] ![d-1] !}{[2][d-i+1] !} \lambda^{i-2} \quad(2 \leq i \leq d) .
\end{aligned}
$$

Proof. In [1] the action of $A$ on the basis of Lemma 4.2 was described. The action of $R$ follows from this action of $A$ and Lemmas 3.2 and 4.3. $\square$

TheOREM 4.6. [1] With reference to Theorem 4.5,

$$
\begin{aligned}
& L v_{i}=v_{i-1} \quad(2 \leq i \leq d), \\
& L v_{1}=\gamma_{d} \mu v_{0}^{*}, \\
& L v_{i}^{*}=\lambda_{d-i} v_{i-1}^{*}+\gamma_{i} \mu v_{i-1} \quad(2 \leq i \leq d-1), \\
& L v_{1}^{*}=\left(\lambda_{d-1}+\gamma_{d-1} \mu^{2}\right) v_{0}^{*}, \\
& L v_{0}^{*}=0 .
\end{aligned}
$$

Proof. In [1] the action of $A^{*}$ on the basis of Lemma 4.2 was described. The action of $L$ follows from this action of $A^{*}$ and Lemmas 3.2 and 4.3.

5. The operators $\ell$ and $r$. In this section we describe the maps $\ell$ and $r$ referred to in the introduction.

TheOREM 5.1. With reference to Theorem 4.5 and Remark 4.4, let $\ell: V \rightarrow V$ be the linear transformation which acts on the basis of Lemma 4.2 as follows.

$$
\begin{aligned}
\ell v_{1} & =\frac{[d-1][d-1] !^{2} \lambda^{d-3} \mu^{2}+[2]^{2} \lambda}{[2] \mu} v_{0}^{*}, \\
\ell v_{i} & =\frac{[d-i]}{[d-i+1] \lambda} v_{i-1}+\frac{[2][d-i] !}{\mu \lambda^{i-2}[d-1] ![i-1] !} \quad(2 \leq i \leq d-1), \\
\ell v_{d} & =\frac{[2]}{[d-1] !^{2} \lambda^{d-2} \mu} v_{d-1}^{*}, \\
\ell v_{0}^{*} & =0 \\
\ell v_{i}^{*} & =[d-i+1][i] v_{i-1}^{*} \quad(1 \leq i \leq d-1) .
\end{aligned}
$$

Then

$$
\begin{aligned}
K \ell & =q^{-2} \ell K, \\
\ell R-R \ell & =\frac{K^{-1}-K}{q-q^{-1}}, \\
\ell L & =L \ell .
\end{aligned}
$$

Proof. Apply each side of Equations (5.1)-(5.3) to the basis in Lemma 4.2. 
TheOREM 5.2. With reference to Theorem 4.5 and Remark 4.4, let $r: V \rightarrow V$ be the linear transformation which acts on the basis of Lemma 4.2 as follows.

$$
\begin{aligned}
r v_{0}^{*} & =\frac{[2]}{[d-1] !^{2} \lambda^{d-2} \mu} v_{1}, \\
r v_{i}^{*} & =\frac{[i]}{[i+1] \lambda} v_{i+1}^{*}+\frac{[2][i] !}{\mu \lambda^{d-i-2}[d-1] ![d-i-1] !} v_{i+1} \quad(1 \leq i \leq d-2), \\
r v_{d-1}^{*} & =\frac{[d-1][d-1] !^{2} \lambda^{d-3} \mu^{2}+[2]^{2} \lambda}{[2] \mu} v_{d}, \\
r v_{d} & =0, \\
r v_{i} & =[d-i][i+1] v_{i+1} \quad(1 \leq i \leq d-1) .
\end{aligned}
$$

Then

$$
\begin{aligned}
K r & =q^{2} r K, \\
r L-L r & =\frac{K-K^{-1}}{q-q^{-1}}, \\
r R & =R r .
\end{aligned}
$$

Proof. Apply each side of Equations (5.4)-(5.6) to the basis in Lemma 4.2.

LEMma 5.3. With reference to Notation 4.1, let $r$ and $\ell$ be the linear transformations of Theorems 5.1 and 5.2 respectively. Then

$$
\begin{aligned}
r^{3} \ell-[3] r^{2} \ell r+[3] r \ell r^{2}-\ell r^{3} & =0, \\
\ell^{3} r-[3] \ell^{2} r \ell+[3] \ell r \ell^{2}-r \ell^{3} & =0 .
\end{aligned}
$$

Proof. Apply each side to the basis in Lemma 4.2.

Proof of Theorem 1.12. Define $\rho: U_{q}\left(\widehat{s l_{2}}\right) \rightarrow \operatorname{End}(V)$ by: $\rho\left(e_{1}^{+}\right)=\ell, \rho\left(e_{0}^{+}\right)=r$, $\rho\left(e_{1}^{-}\right)=R, \rho\left(e_{0}^{-}\right)=L, \rho\left(K_{0}\right)=K$ and $\rho\left(K_{1}\right)=K^{-1}$. Now $\rho$ is a homomorphism by Corollary 2.8, Lemma 3.3, Theorems 5.1 and 5.2, and Lemma 5.3. Moreover, by assumption, $V$ is an irreducible module for $A, A^{*}$, hence $V$ is an irreducible module with respect to $R, L, K$. Hence, Theorem 1.12 follows.

\section{REFERENCES}

[1] H. Alnajjar and B. Curtin. A family of tridiagonal pairs. Linear Algebra Appl., 390:369-384, 2004 .

[2] V. Chari and A. Pressley. Quantum affine algebras. Comm. Math. Phys., 142(2):261-283, 1991.

[3] V. Chari and A. Pressley. Quantum affine algebras and their representations. In Representations of groups (Banff, AB, 1994), volume 16 of CMS Conf. Proc., pp. 59-78. Amer. Math. Soc., Providence, RI, 1995.

[4] S. Hobart and T. Ito. The structure of nonthin irreducible T-modules of endpoint 1: Ladder bases and classical parameters. J. Algebraic Combin., 7(1):53-75, 1998. 
[5] T. Ito, K. Tanabe and P. Terwilliger. Some algebra related to P- and Q-polynomial association schemes. Codes and Association Schemes (Piscataway NJ, 1999), Amer. Math. Soc., Providence RI, 56:167-192, 2001

[6] T. Ito and P. Terwilliger. The shape of a tridiagonal pair. J. Pure Appl. Algebra, 188(1-3):145$160,2004$.

[7] T. Ito and P. Terwilliger. Tridiagonal pairs and the quantum affine algebra $U_{q}\left(\widehat{s l_{2}}\right)$. Preprint.

[8] P. Terwilliger. Two linear transformations each tridiagonal with respect to an eigenbasis of the other. Linear Algebra Appl., 330:149-203, 2001.

[9] P. Terwilliger. Two relations that generalize the q-Serre relations and Dolan-Grady relations. Physics and combinatorics 1999 (Nagoya). World Scientific Publishing, River Edge, NJ, 2001

[10] P. Terwilliger. Leonard pairs from 24 points of view. Rocky Mountain J. Math., 32(2):827-888, 2002.

[11] P. Terwilliger. Two linear transformations each tridiagonal with respect to an eigenbasis of the other: the $T D-D$ and the $L B-U B$ canonical form. Preprint.

[12] P. Terwilliger. Introduction to Leonard pairs. OPSFA Rome 2001. J. Comput. Appl. Math., 153(2):463-475, 2003. 